\title{
Increasing the efficiency of organic solar cells using dielectric spherical nanoparticles
}

\author{
Yu.V. Vladimirova ${ }^{1, *}$, and V.N. Zadkov ${ }^{1,2,3}$ \\ ${ }^{1}$ International Laser Center and Faculty of Physics, Lomonosov State University, 119991 Moscow, \\ Russia \\ ${ }^{2}$ Kolmogorov School, Lomonosov State University, 119991 Moscow, Russia \\ ${ }^{3}$ Institute for Spectroscopy RAS, 108840 Troitsk, Moscow, Russia
}

\begin{abstract}
In this paper we review recent progress in using plasmonic nanoparticles for improving efficiency of the organic photovoltaic (PV) cells with embedded plasmonic nanoparticles. Specifically, we discuss how the plasmonic nanoparticles can be used for guiding and concentrating the light for enhanced absorption, paying attention to both fundamentals and design considerations, as well as to realization of the broadband plasmonic scattering to better utilize the incoming solar spectrum. Plus to that, we discuss potential advantages of using dielectric nanoparticles in PV solar cells.
\end{abstract}

The development of alternative sources of energy such as solar photovoltaic (PV) devices is one of the urgent problems of our society. In this field primary tasks are the increase of the solar cells efficiency and significant reduction in their price. For the crystalline silicon solar cells, the problem of increasing their efficacy was largely solved, however they are still very expensive. By contrast, organic solar cells are rather cheap in production, but have quite low efficiency so far [1]. That is why the development of various methods that allow to increase the organic solar cells efficiency is of prime importance [2].

One option to raise an efficiency of a PV solar cell is the use of plasmonic nanoparticles, which are embedded into the solar cell. Each nanoparticle serves then as a nanoantenna concentrating the incident electromagnetic field in the absorption area and thereby increasing the efficiency of the solar cell [3]. Such nanostructures are already used in silicon solar cells, but technologies of the nanostructure deposition are very expensive and laborious.

We discuss here the potential advantages of using dielectric nanoparticles in organic PV cells. High-refractive index dielectric nanoparticles may exhibit strong directional forward light scattering at visible and near-infrared wavelengths due to interference of simultaneously excited electric and magnetic dipole resonances [4,5]. For a spherical particle shape, the so-called first Kerker's condition can be realized, at which the backward scattering practically vanishes for some relation between the refractive index and the particle's size.

\footnotetext{
${ }^{*}$ Corresponding author: yu.vladimirova@physics.msu.ru
} 
Experimentally, directional light scattering by spherical silicon nanoparticles in the visible spectral range was demonstrated, for example, in Ref. [6]. Many investigators considered scattering properties of a silicon nanoparticle in free space, but in the real applications the nanoparticles are often located near a substrate and the influence of the substrate may be very significant [5-7]. In this work, we derived optimal conditions for forward light scattering by spherical homogeneous dielectric nanoparticles with high refractive index in non-magnetic and non-absorbing media near a dielectric substrate.

We consider substrates with $n=1.5$ (P3HT:PCBM). Our calculations of the scattering diagram of a nanoparticle located near a dielectric substrate demonstrate its strong anisotropy in both forward and backward directions. We studied in detail how the scattering diagram of a spherical silicon nanoparticle near a dielectric substrate depends on the radius of the nanoparticle and distance to the substrate, as well as on the polarization, wavelength and angle of the incident light wave [8].
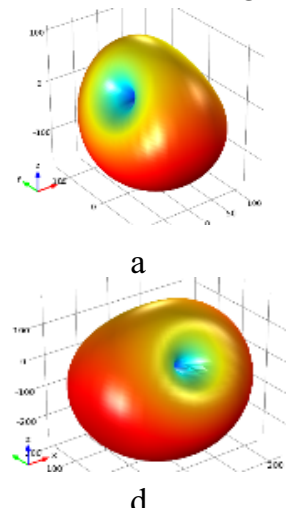

d

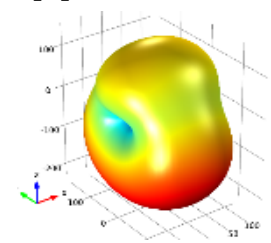

$\mathrm{b}$

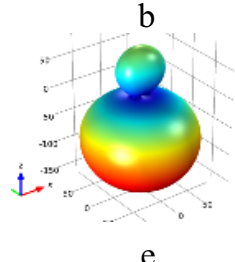

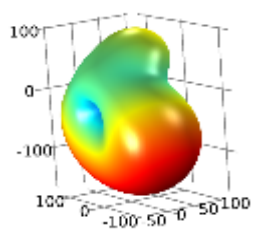

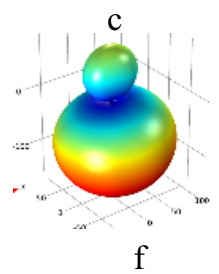

Fig. 1. Scattering diagrams of a silicon nanoparticle with a radius of $65 \mathrm{~nm}$ in free space (a,d and near a substrate $(n=1.5)$ for normal incidence $\theta=0(\mathrm{~b}, \mathrm{e})$ and $\theta=\pi / 6(\mathrm{c}, \mathrm{f})$ for the incidence radiation wavelength $\lambda=550 \mathrm{~nm}(\mathrm{a}, \mathrm{b}, \mathrm{c})$ and $650 \mathrm{~nm}(\mathrm{~d}, \mathrm{e}, \mathrm{f})$.

Scattering diagrams of a silicon nanoparticle with a radius of $65 \mathrm{~nm}$ in free space (a,d) and near a substrate $(n=1.5)$ for normal incidence $\theta=0(\mathrm{~b}, \mathrm{e})$ and $\theta=\pi / 6(\mathrm{c}, \mathrm{f})$ for the incidence radiation wavelength $\lambda=550 \mathrm{~nm}(\mathrm{a}, \mathrm{b}, \mathrm{c})$ and $650 \mathrm{~nm}(\mathrm{~d}, \mathrm{e}, \mathrm{f})$ is presented in the figure. From this figure it follows that the maximum forward scattering for such silicon nanoparticle is observed for $\lambda=350,460,540,640 \mathrm{~nm}$ for normal incidence $\theta=0$ and $\theta=\pi / 6$.

The authors would like to acknowledge financial support from the Russian Foundation for Basic Research under the grant No. 16-02-00816.

\section{References}

1. M. Gu, Z. Ouyang, J. Baohua et al., Nanophotonics 1, 235 (2012)

2. Z. Tang, W. Tress, O. Inganäs, Materials today 17, 389 (2014)

3. L. Novotny, B. Hecht, Principles of Nano-Optics, (Cambridge University Press, 2012).

4. Y.H. Fu, A.I. Kuznetsov, A.E. Miroshnichenko et al., Nat. Commun. 4, 1527 (2013)

5. M.I. Tribelsky, J.-M. Geffrin, A. Litman, Ch. Eyraud, F. Moreno, Sci. Reports 5, 12288 (2015)

6. A. Pors, S.K.H. Andersen, S.I. Bozhevolnyi, Opt. Express 23, 28808 (2015)

7. A.E. Miroshnichenko, A.B. Evlyukhin, Y.S. Kivshar, B.N. Chichkov, ACS Photonics 2, 1423 (2015) 